\title{
APPLICATIONS OF A NEW $K$-THEORETIC THEOREM TO SOLUBLE GROUP RINGS
}

\author{
P. H. KROPHOLLER, P. A. LINNELL AND J. A. MOODY
}

(Communicated by Donald S. Passman)

\begin{abstract}
Let $R$ be a ring and let $G$ be a soluble group. In this situation we shall give necessary and sufficient conditions for $R G$ to have a right Artinian right quotient ring. In the course of this work, we shall also consider the Goldie rank problem for soluble groups and record an affirmative answer to the zero divisor conjecture for soluble groups.
\end{abstract}

1. Introduction. We shall start by describing the theorem referred to in the title. Let $R$ be a ring (all rings in this paper will have a one), let $G$ be a group, and let $R * G$ denote a crossed product (see [11]). Thus $R * G$ may be viewed as a free $R$-module with basis $\{\bar{g} \mid g \in G\}$ where each $\bar{g}$ is a unit in $R * G$, and ring structure satisfying $R \bar{g} R \bar{h}=R \overline{g h}$. Of course, $R * G$ is not uniquely determined by $R$ and $G$. However if $H \leq G$, then $R * H$ is defined to be $\bigoplus_{h \in H} R \bar{h}$, a subring of $R * G$. Another way of describing $R * G$ is that it is a $G$-graded ring (or group-graded ring) with a unit in each degree $[5, \S 5]$.

For a right Noetherian ring $S$, let $G_{0}(S)$ denote the Grothendieck group associated with the category of all finitely generated right $S$-modules. If $R$ is right Noetherian and $G$ is polycyclic-by-finite, then $R * G$ is also right Noetherian. Moreover if $H \leq G$, then the functor $M \mapsto M \otimes_{R * H} R * G$ preserves exact sequences and hence induces a natural group homomorphism $G_{0}(R * H) \rightarrow G_{0}(R * G)$ which is called the induction map. The following theorem was proved by the third author in his thesis [7] and described in [8] (see [9] for full details).

THEOREM 1.1. Let $R$ be a right Noetherian ring, let $G$ be a polycyclic-by-finite group, and let $\mathscr{F}(G)$ denote the set of finite subgroups of $G$. Then the natural (induction) map $\bigoplus_{F \in \mathscr{F}(G)} G_{0}(R * F) \rightarrow G_{0}(R * G)$ is surjective.

It is well known that such a theorem would have important consequences in algebra, and the purpose of this paper is to consider a few of these in the theory of group rings of soluble groups.

Ever since the papers of Brown [2] and Farkas and Snider [6], it has been folklore that the zero divisor conjecture for soluble-by-finite groups (cf. Theorem 1.4) would follow from Theorem 1.1, but this fact has never been published because it was never expected that such a theorem would be proved. An indication of how to deduce

Received by the editors April 20, 1987 and, in revised form, October 26, 1987.

1980 Mathematics Subject Classification (1985 Revision). Primary 16A08, 16A27; Secondary $19 \mathrm{M} 05$.

Key words and phrases. Group ring, zero divisor conjecture, Goldie rank, quotient ring, Grothendieck group. 
the zero divisor conjecture for soluble-by-finite groups from Theorem 1.1 is given in [8].

It was first recorded by Rosset [12] that the special case of Theorem 1.1 when $R * G$ is an ordinary group algebra with coefficients in a commutative field ("strong conjecture (B)" in the terminology of [12]) is already sufficient to establish the Goldie rank conjecture for polycyclic-by-finite groups (see further on in the introduction for a description of this problem). This consequence is described in [ 7 and 8].

To describe the results of this paper precisely, we need to establish some notation. Let $S \subseteq Q$ be rings. Following pp. 19-20 of [1], we say that $Q$ is a right quotient ring for $S$ provided that every regular element (i.e. nonzero divisor) of $S$ is a unit in $Q$, and every element of $Q$ is of the form $a c^{-1}$ for some elements $a$ and $c$ of $S$ with $c$ regular. In general $S$ need not possess a right quotient ring. However when such a $Q$ exists, it is uniquely determined by $S$. Also, we shall let $\mathscr{C}$ denote the class of elementary amenable groups (see [4]). This is the smallest class of groups which

(i) contains all abelian and all finite groups,

(ii) is extension closed,

(iii) is closed under directed unions.

Clearly $\mathscr{C}$ contains all soluble-by-finite groups.

A problem closely related to the Goldie rank conjecture is that of when a group ring has a right Artinian right quotient ring. Progress on this (without the aid of Theorem 1.1) has been made in [3]. The main result of this paper is

THEOREM 1.2. Let $R$ be a ring and let $G \in \mathscr{C}$. Then $R G$ has a right Artinian right quotient ring if and only if $R$ has a right Artinian right quotient ring and the finite subgroups of $G$ have bounded order.

The reader will find that the proof of this theorem cannot be significantly shortened by restricting attention to soluble-by-finite groups.

Now suppose $k$ is a division ring, $G \in \mathscr{C}, \Delta^{+}(G)=1$ (i.e. $G$ has no nontrivial finite normal subgroup), and the finite subgroups of $G$ have bounded order. Then $k G$ has a right Artinian right quotient ring by Theorem 1.2 and $k G$ is prime by Theorem 2 on p. 37 of [11]. It follows that $k G$ has a right quotient ring which is an $l \times l$ matrix ring over a division ring for some integer $l$. In the special case that $G$ is polycyclic-by-finite, the Goldie rank conjecture states that $l$ is the l.c.m of the orders of the finite subgroups of $G$; see pp. 19-21 of [11] for further information concerning this problem. As a by-product of the proof of Theorem 1.2, we obtain the following result.

THEOREM 1.3. Let $k$ be a division ring, let $G \in \mathscr{C}$, and assume that $\Delta^{+}(G)=$ 1. Suppose the finite subgroups of $G$ have bounded order, and write l for the l.c.m. of the orders of the finite subgroups of $G$. Then $k G$ has a right quotient ring which is an $l \times l$ matrix ring over a division ring.

For completeness, we record the following immediate consequence.

THEOREM 1.4. Let $k$ be a division ring and let $G \in \mathscr{C}$. If $G$ is torsion-free, then $k G$ is a domain.

We do not know whether Theorem 1.4 remains true for arbitrary domains $k$. 
We conclude this paper with a brief discussion on how to extend Theorem 1.3 to some semiprime crossed products.

Part of this work was done while the second author was at Stuttgart University. He is deeply grateful to Klaus Roggenkamp and the other mathematicians there for their warm hospitality. He would also like to thank the Alexander von HumboldtStiftung for financial support.

2. Notation and assumed results. As usual, $\mathbb{Q}$ will denote the rational numbers, $\mathbb{C}$ the complex numbers, and $\mathbf{P}$ the positive integers $\{1,2,3, \ldots\}$. For any group $G$ and $Y \subseteq G$, the subgroup generated by the finite normal subgroups of $G$ will be denoted by $\Delta^{+}(G)$ (see p. 2 of [11] or p. 81 of [10]), the set of finite subgroups of $G$ will be denoted by $\mathscr{F}(G)$, and $\langle Y\rangle$ will indicate the subgroup generated by $Y$. If $q_{1}, \ldots, q_{n} \in \mathbb{Q} \backslash\{0\}$ and $l \in \mathbf{P}$, then $l=$ l.c.m. $\left\{q_{1}, \ldots, q_{n}\right\}$ means $\langle 1 / l\rangle=\left\langle 1 / q_{1}, \ldots, 1 / q_{n}\right\rangle$; in the special case when the $q_{i}$ are nonzero integers, this coincides with the usual definition of l.c.m. Let $R$ be a ring, $X \subseteq R, A \leq G$, $B \in \mathscr{F}(G)$, and $g \in G$. Then we write $r_{R}(X)=\{y \in R \mid x y=0$ for all $x \in X\}$, $A^{g}=g^{-1} A g$, and $|B|$ for the order of $B$. If $S$ is a multiplicatively closed subset of $R$ and $R$ satisfies the right Ore condition with respect to $S$ (see p. 21 of [1]), then we can form the ring $R S^{-1}$, which consists of elements $r s^{-1}$ with $r \in R$ and $s \in S$. When $A$ has finite index in $G$, then $G: A$ will indicate this index. If $\mathscr{X}$ and $\mathscr{Y}$ are classes of groups, then $G \in L \mathscr{X}$ will mean that every finite subset of $G$ is contained in some $\mathscr{X}$-subgroup of $G$, and $G \in \mathscr{X} Y$ will mean that there exists $H \triangleleft G$ such that $H \in \mathscr{X}$ and $G / H \in \mathscr{Y}$. The following three results are well known.

LEMMA 2.1. Let $R$ be a ring and let $G$ be a finitely generated abelian-by-finite group. If $R * G$ is prime and $R$ has a right Artinian right quotient ring, then $R * G$ has a simple Artinian right quotient ring.

PROOF. Let $T$ be the set of regular elements of $R$, so that $R T^{-1}$ is a right Artinian ring. Then the rings $R T^{-1} * G$ and $(R * G) T^{-1}$ exist, are prime, and are isomorphic. Since $R T^{-1} * G$ is a prime right Noetherian ring, it follows from Goldie's theorem (Theorem 1.28 of [1]) that it has a simple Artinian right quotient ring, as required.

LEMMA 2.2. Let $R$ be a right Noetherian ring and let $S$ be a multiplicatively closed subset of $R$ such that the ring $R S^{-1}$ exists. Then the natural (induction) map $G_{0}(R) \rightarrow G_{0}\left(R S^{-1}\right)$ is surjective.

PROOF. This is because every finitely generated $R S^{-1}$-module is of the form $N \otimes_{R} R S^{-1}$ for some finitely generated $R$-module $N$.

Suppose $R$ has a simple Artinian right quotient ring $Q=M_{n}(D)$ for some $n \in \mathbf{P}$ and division ring $D$. If $M$ is a finitely generated $R$-module, then we define $\rho_{R}[M]=\operatorname{dim}_{D}\left[M \otimes_{R} Q\right] / n^{2}$ and when $R$ is right Noetherian, then $\rho_{R}$ induces a homomorphism $\bar{\rho}_{R}: G_{0}(R) \rightarrow \mathbb{Q}$. Note that the image of $\bar{\rho}_{R}$ is $\langle 1 / n\rangle$. Evidently if $\psi: G_{0}(Q) \rightarrow \mathbb{Q}$ is a homomorphism such that $\psi[Q]=1$, then $\psi=\bar{\rho}_{Q}$. The following lemma is elementary.

LEMMA 2.3. Let $R \subseteq T$ be rings and let $H \triangleleft G$ be groups.

(i) Suppose $R$ and $T$ have simple Artinian right quotient rings $U$ and $V$ respectively such that $U \subseteq V$. If $M$ is a finitely generated $R$-module, then $\rho_{T}\left[M \otimes_{R} T\right]=$ $\rho_{R}[M]$. 
(ii) Suppose $H$ has finite index in $G$, and $R * H$ and $R * G$ have simple Artinian right quotient rings. If $M$ is a finitely generated $R * G$-module, then $(G: H) \rho_{R * G}[M]=\rho_{R * H}[M]$.

ProOF. (i) We may assume that $R$ and $T$ are simple Artinian rings. If $M$ is a finitely generated $R$-module, then the map $M \mapsto \rho_{T}\left[M \otimes_{R} T\right]$ induces a map $\psi: G_{0}(R) \rightarrow \mathbb{Q}$. But $\rho_{T}\left[R \otimes_{R} T\right]=\rho_{T}[T]=1$, hence $\psi=\bar{\rho}_{R}$ and the result follows.

(ii) Let $Q$ and $W$ be the simple Artinian right quotient rings of $R * H$ and $R * G$ respectively, and let $S$ be the set of nonzero divisors of $R * H$. Since $Q \cong$ $(R * H) S^{-1}$, we see that $(R * G) S^{-1}$ is Artinian, hence $W \cong(R * G) S^{-1}$ and we deduce that $W$ is a free $Q$-module of $\operatorname{rank} G: H$. Therefore the map $\psi: G_{0}(W) \rightarrow \mathbb{Q}$ defined by $\psi[N]=\bar{\rho}_{Q}[N] / G: H$ satisfies $\psi(W)=1$, hence $\psi=\bar{\rho}_{W}$ and we conclude that $\rho_{R * H}[M]=(G: H) \rho_{R * G}[M]$, as required.

We can extend $\rho$ to more general rings as follows. Let $R$ be a ring, let $N$ be the nilradical of $R$, let $s \in \mathbb{P}$ and let $M$ be an $R$-module. Suppose $N^{s}=0$ and $R / N$ is right Goldie $[\mathbf{1}$, p. 8]. Then $R / N$ has a semisimple Artinian right quotient ring $Q\left[1\right.$, Theorem 1.27]. Thus if $L$ is an $R / N$-module, we can define $\rho_{R}(L)$ to be the length of $L \otimes_{R} Q$ as a right $Q$-module (in the case $L$ is finitely generated, $N=0$ and $Q$ is simple, this coincides with the definition of $\rho$ just before Lemma 2.3). Then $\rho$ can be extended to all $R$-modules by defining

$$
\rho_{R}(M)=\sum_{i=1}^{s} \rho_{R}\left(M N^{i-1} / M N^{i}\right) .
$$

The same proof as that of Theorem 2.2 of [1] shows

LEMMA 2.4. (a) If $K$ is a submodule of $M$, then $\rho_{R}(M)=\rho_{R}(K)+\rho_{R}(M / K)$.

(b) $\rho_{R}(M)=0$ if and only if for each $m \in M$, there exists $\alpha \in R$ such that its image in $R / N$ is a nonzero divisor and $m \alpha=0$.

By following the proof of Theorem 2.3 of [1] we now have (we would like to thank K. A. Brown for pointing out that our original formulation of this lemma was incorrect)

LEMMA 2.5. Assume that whenever $\alpha \in R$ is such that its image in $R / N$ is a nonzero divisor, then $\alpha$ is a nonzero divisor. If $\rho_{R}(R)<\infty$, then $R$ has a right Artinian right quotient ring.

3. Group theoretic results. Our first task is to give an alternative description for the class of groups $\mathscr{C}$. Let $\mathscr{B}$ denote the class of all finitely generated abelianby-finite groups and for each ordinal $\alpha$, define $\mathscr{X}_{\alpha}$ inductively as follows:

$$
\begin{aligned}
& \mathscr{X}_{0}=\{1\} \\
& \mathscr{X}_{\alpha}=\left(L \mathscr{X}_{\alpha-1}\right) \mathscr{B} \text { if } \alpha \text { is a successor ordinal, } \\
& \mathscr{X}_{\alpha}=\bigcup_{\beta<\alpha} \mathscr{X}_{\beta} \text { if } \alpha \text { is a limit ordinal. }
\end{aligned}
$$

Setting $\mathscr{X}=\bigcup_{\alpha \geq 0} \mathscr{X}_{\alpha}$, we can now state the following lemma. 
LEMMA 3.1. (i) $\mathscr{X}=\mathscr{C}$.

(ii) Each $\mathscr{X}_{\alpha}$ is subgroup closed.

ProOF. Clearly $\mathscr{X} \subseteq \mathscr{C}, \mathscr{X}$ contains every abelian group and every finite group, and $\mathscr{Z}$ is closed under directed unions. Also it is easy to show by induction on $\alpha$ that each $\mathscr{X}_{\alpha}$ is subgroup closed. Therefore we need to prove that $\mathscr{X}$ is extension closed.

We show by induction on $\beta$ that $\mathscr{X}_{\alpha} \mathscr{X}_{\beta} \subseteq \mathscr{X}_{\alpha+\beta}$; the case $\beta=0$ is obvious. If $\beta=\gamma+1$ for some ordinal $\gamma$, then

$$
\begin{aligned}
\mathscr{X}_{\alpha} \mathscr{X}_{\beta} & =\mathscr{X}_{\alpha}\left(\left(L \mathscr{X}_{\gamma}\right) \mathscr{B}\right) \subseteq\left(\mathscr{X}_{\alpha}\left(L \mathscr{X}_{\gamma}\right)\right) \mathscr{B} \subseteq\left(L\left(\mathscr{X}_{\alpha} \mathscr{X}_{\gamma}\right)\right) \mathscr{B} \\
& \subseteq\left(L \mathscr{X}_{\alpha+\gamma}\right) \mathscr{B} \quad \text { (by induction) } \\
& =\mathscr{X}_{\alpha+\beta} .
\end{aligned}
$$

On the other hand if $\beta$ is a limit ordinal, then $\mathscr{X}_{\beta}=\bigcup_{\gamma<\beta} \mathscr{X}_{\gamma}$ and

$$
\begin{aligned}
\mathscr{X}_{\alpha} \mathscr{X}_{\beta} & =\mathscr{X}_{\alpha}\left(\bigcup_{\gamma<\beta} \mathscr{X}_{\gamma}\right)=\bigcup_{\gamma<\beta} \mathscr{X}_{\alpha} \mathscr{X}_{\gamma} \\
& \subseteq \bigcup_{\gamma<\beta} \mathscr{X}_{\alpha+\gamma} \quad \text { (by induction) } \\
& \subseteq \mathscr{X}_{\alpha+\beta}
\end{aligned}
$$

as required.

Lemma 3.1 allows us to associate to each group $G \in \mathscr{C}$, the least ordinal $\alpha$ such that $G \in \mathscr{X}_{\alpha}$. Our results are proved by transfinite induction on this ordinal. Note that every soluble-by-finite group belongs to $\mathscr{X}_{\omega}$ (where $\omega$ is the least infinite ordinal), thus if one is only interested in results for soluble-by-finite groups, then transfinite induction could be replaced by ordinary induction. However this would not simplify any of the arguments, so we prove the results in the general case.

LEMMA 3.2. If $G$ is a group with no infinite locally finite subgroup, then $\Delta^{+}(G)$ is finite.

Proof. Since $\Delta^{+}(G)$ is locally finite (use Lemma 7 (i) on p. 9 of [11]), the result is clear.

LEMMA 3.3. Let $G$ be a group with no infinite locally finite subgroup. If $\Delta^{+}(G)=1$, then $G$ has a finitely generated subgroup $P$ such that whenever $P \leq$ $B \leq G$, then $\Delta^{+}(B)=1$.

ProOF. Let $F$ be a maximal finite subgroup of $G$ and for each $1<H \leq F$, choose $g_{H} \in G \backslash N_{G}(H)$. Then $P=\left\langle F, g_{H} \mid 1<H \leq F\right\rangle$ clearly has the required properties.

\section{Proof of Theorems 1.2 and 1.3 .}

LEMMA 4.1. Let $H \triangleleft G \in \mathscr{C}$, let $n \in \mathbf{P}$ and let $R$ be a simple Artinian ring. Suppose $|G / H|<\infty$, the orders of the finite subgroups of $G$ are bounded and $\Delta^{+}(H)=1$. Then

(i) $R * H$ has a simple Artinian right quotient ring $(R * H) S^{-1}$ where $S$ is the set of nonzero divisors of $R * H$. 
(ii) The natural map $\bigoplus_{F \in \mathscr{F}(G)} G_{0}(R * F) \rightarrow G_{0}\left(R * G S^{-1}\right)$ is surjective.

(iii) If $F \in \mathscr{F}(H)$ and $M$ is a finitely generated $R * F$-module, then

$$
|F| \rho_{R * H}\left[M \otimes_{R * F} R * H\right]=\rho_{R}[M] .
$$

(iv) If $m=$ l.c.m. $\left\{|F| / \rho_{R}[M] \mid F \in \mathscr{F}(H), M \neq 0\right.$ is a finitely generated $R * F$ module $\}$, then $(R * H) S^{-1}$ is an $m \times m$ matrix ring over a division ring.

PROOF. The result will be proved by transfinite induction, so by Lemma 3.1 choose the least ordinal $\alpha$ such that $H \in \mathscr{X}_{\alpha}$, and assume that the result is true whenever $H \in \mathscr{X}_{\beta}$ and $\beta<\alpha$. Let $F \in \mathscr{F}(H)$, let $M$ be a finitely generated $R * F$-module, and let $l$ be the l.c.m. of $\left\{|E| / \rho_{R}[N] \mid E \in \mathscr{F}(H), N \neq 0\right.$ is a finitely generated $R * E$-module\}.

Now $\alpha$ cannot be a limit ordinal, and the result is clearly true if $\alpha=0$. Therefore we may assume that $\alpha=\gamma+1$ for some ordinal $\gamma$. First suppose $H \in L \mathscr{X}_{\gamma}$. By Lemma 3.3, there exists a finitely generated subgroup $P$ of $H$ such that whenever $P \leq P_{1} \leq H$, then $\Delta^{+}\left(P_{1}\right)=1$. Let $\left\{G_{i} \mid i \in \mathscr{I}\right\}$ be the family of finitely generated subgroups of $G$ containing $P$ and for each $i \in \mathcal{I}$, let $H_{i}=H \cap G_{i}$, and let $S_{i}=S \cap\left(R * H_{i}\right)$, the set of nonzero divisors of $R * H_{i}$. Then $\Delta^{+}\left(H_{i}\right)=1$ and since $H_{i} \in \mathscr{X}_{\gamma}$ by Lemma 3.1(ii), we can form the simple Artinian right quotient ring $Q_{i}=\left(R * H_{i}\right) S_{i}^{-1}$ for all $i \in \mathscr{J}$, and it follows that we can form the $\operatorname{ring} Q=$ $(R * H) S^{-1}$. Using (iv), we see that $Q_{i}$ is a $d_{i} \times d_{i}$ matrix ring over a division ring for some $d_{i} \leq l$. Since $Q=\bigcup_{i \in \mathcal{I}} Q_{i}$, we see that if $I_{0}>I_{1}>\cdots>I_{n}$ is a strictly descending sequence of right ideals in $Q$, then $I_{0} \cap Q_{i}>I_{1} \cap Q_{i}>\cdots>I_{n} \cap Q_{i}$ is a strictly descending sequence of right ideals in $Q_{i}$ for some $i \in \mathscr{I}$, hence $n \leq l$ and we deduce that $Q$ is Artinian. This proves (i). Since $(R * G) S^{-1}=\bigcup_{i \in \mathcal{I}}\left(R * G_{i}\right) S_{i}^{-1}$, we see that $G_{0}\left(R * G S^{-1}\right)=\operatorname{Lim} G_{0}\left(R * G_{i} S_{i}^{-1}\right)$ and (ii) follows easily. Next choose $i$ such that $F \leq H_{i}$. Then $\hat{\rho}_{R * H_{i}}[N]=\rho_{R * H}\left[N \otimes_{R * H_{i}} R * H\right]$ for all finitely generated $R * H_{i}$-modules $N$ by Lemma 2.3(i), and (iii) is clear. Finally (iv) follows from (i), (ii), and (iii).

Now suppose there exists $A \triangleleft H$ such that $H / A \in \mathscr{B}$ and $A \in L \mathscr{X}_{\gamma}$. Write $B=\bigcap_{g \in G} A^{g}$ and $T=S \cap(R * B)$, the set of nonzero divisors of $R * B$. Note that $B \triangleleft G$ and $G / B \in \mathscr{B}$. Since $B \in L \mathscr{X}_{\gamma}$ by Lemma 3.1 (ii) and $\Delta^{+}(B)=1$, we see from the previous paragraph that the ring $K=(R * B) T^{-1}$ exists and is simple Artinian. Observing that $R * H \cong(R * B) * H / B$ (see pp. 29-30 of [11]) and that $R * H$ is prime (see Theorem 2 on p. 37 of [11]), application of Lemma 2.1 now yields (i). By Theorem 1.1, the natural map

$$
\bigoplus_{E \in \mathscr{F}(G / B)} G_{0}(K * E) \rightarrow G_{0}(K * G / B)
$$

is surjective. But

$$
G_{0}(K * G / B)=G_{0}\left((R * G) T^{-1}\right) \rightarrow G_{0}\left((R * G) S^{-1}\right)
$$

is also surjective by Lemma 2.2 , and (ii) is verified. Finally let $C / B$ be a torsionfree normal abelian subgroup of finite index in $H / B$. Since $\Delta^{+}(H)=1$, we see that $\Delta^{+}(C)=1$ and hence $R * C$ has a simple Artinian right quotient ring by (i). If $D=F \cap C$, then $M \otimes_{R * F} R * C F \cong M \otimes_{R * D} R * C$ as $R * C$-modules and 
$D \subseteq B$. Therefore

$$
\begin{aligned}
\rho_{R * H}\left[M \otimes_{R * F} R * H\right] & =\rho_{R * C F}\left[M \otimes_{R * F} R * C F\right] \quad \text { by Lemma 2.3(i) } \\
& =\frac{1}{C F: C} \rho_{R * C}\left[M \otimes_{R * F} R * C F\right] \quad \text { by Lemma 2.3(ii) } \\
& =\frac{1}{F: D} \rho_{R * C}\left[M \otimes_{R * D} R * C\right] \\
& =\frac{1}{F: D} \rho_{R * B}\left[M \otimes_{R * D} R * B\right] \quad \text { by Lemma 2.3(i) } \\
& =\frac{1}{|F|} \rho_{R}[M] \quad \text { by (iii) for } B .
\end{aligned}
$$

This establishes (iii), and (iv) now follows from (i), (ii) and (iii).

PROPOSITION 4.2. Let $R$ be a ring, let $G \in \mathscr{C}$ and let $R * G$ be a crossed product. If $R$ has a right Artinian right quotient ring and the finite subgroups of $G$ have bounded order, then $R * G$ has a right Artinian right quotient ring.

ProOF. Clearly we may assume that $R$ is right Artinian. Let $H=\Delta^{+}(G)$. Since $H$ is finite by Lemma 3.2 and $R * G=(R * H) * G / H$ (see pp. 29-30 of [11]), we may assume that $\Delta^{+}(G)=1$. Now $R$ has only finitely many maximal two-sided ideals and the conjugation action of $G$ on $R$ permutes these ideals, hence a normal subgroup $S$ of finite index stabilizes these ideals. If $R * S$ has a right Artinian right quotient ring and $T$ is the set of regular elements of $R * S$, then we can form the rings $(R * S) T^{-1}$ and $(R * G) T^{-1}$. Since $(R * S) T^{-1}$ is right Artinian, we see that $(R * G) T^{-1}$ is right Artinian and hence $R * G$ has a right Artinian right quotient ring. Moreover $\Delta^{+}(S)=1$ so by replacing $G$ with $S$, we may assume that $G$ stabilizes the maximal ideals of $R$. Let $N$ be the radical of $R$ and write $R / N=R_{1} \oplus R_{2} \oplus \cdots \oplus R_{n}$ where the $R_{i}$ are simple Artinian rings. Then

$$
R * G / N R * G \cong(R / N) * G \cong R_{1} * G \oplus R_{2} * G \oplus \cdots \oplus R_{n} * G
$$

and therefore has a semisimple Artinian right quotient ring by Lemma 4.1(i). Furthermore $N R * G$ is nilpotent, and $N^{i-1} R * G / N^{i} R * G$ is a finitely generated $R * G / N R * G$-module for $i \in \mathbf{P}$ because $N^{i-1} / N^{i}$ is a finitely generated $R$-module, so $\rho_{R * G}(R * G)<\infty$ by Lemma 2.4 (a). Thus in view of Lemma 2.5, it remains to prove that if $\alpha \in R * G$ is such that its image in $R / N * G$ is a nonzero divisor, then $\alpha$ is a nonzero divisor in $R * G$.

suppose $0 \neq \beta \in R * G$. Then there exists $r \in \mathbf{P}$ such that $\beta \in$ $N^{r-1}(R * G) \backslash N^{r}(R * G)$. Choose $J \triangleleft_{l} R$ such that $N^{r} \subset J \subseteq N^{r-1}, J / N^{r}$ is finitely generated as a left $R$-module and $\beta \in(R * G) J$. Then $J / N^{r}$ is a finitely generated left $R / N$-module, hence there exists $I \triangleleft_{l} R$ such that $N^{r} \subseteq I \subset J, I$ is maximal in $J$ and $\beta \notin(R * G) I$. Since $J / I$ is isomorphic to a minimal left ideal of $R / N$, it follows that $(R * G) J /(R * G) I$ is isomorphic to an $R / N * G$-submodule of $R / N * G$ and hence $\alpha \beta \neq 0$. A similar but slightly easier argument shows that $\beta \alpha \neq 0$ and so $\alpha$ is a nonzero divisor as required.

LEMMA 4.3. Let $R$ be a ring and let $H \triangleleft G$ be groups with $H$ finite. If $R G$ has a right Artinian right quotient ring, then so does $R[G / H]$. 
ProOF. Let $Q$ be the right Artinian right quotient ring of $R G$, let $I$ be the augmentation ideal of $R H$, and suppose $\alpha \in I Q \cap R G$. Write

$$
e=\sum_{h \in H} h \text { and } \alpha=\sum_{i=1}^{n} \alpha_{i} t_{i}
$$

where $\alpha_{i} \in R H, t_{i} \in G$ and $H t_{i} \neq H t_{j}$ for $i \neq j$. Then $e \alpha=0$, hence $e \alpha_{i}=0$ and it follows that $\alpha_{i} \in I$ for $i=1,2, \ldots, n$. Therefore $I Q \cap R G=I R G$ and since $I Q$ is a two-sided ideal of $Q$ by Theorem 1.31 of [1], we deduce that $Q / I Q$ is a right Artinian right quotient ring for $R[G / H]$.

LEMMA 4.4. Let $R$ be a ring, let $G$ be a group and let $Q$ be a right Artinian ring containing $R G$. Then $G$ has no infinite locally finite subgroup.

PROOF. If $G$ has an infinite locally finite subgroup, then there exists a strictly ascending sequence of finite subgroups of $G, H_{1}<H_{2}<H_{3}<\cdots$. For $i \in \mathbf{P}$ let $e_{i}=\sum_{h \in H_{i}} h$ and let $I_{i}$ be the augmentation ideal of $R H_{i}$. Then $r_{Q}\left(I_{1}\right) \supseteq$ $r_{Q}\left(I_{2}\right) \supseteq \cdots$ and $e_{i} \in r_{Q}\left(I_{i}\right) \backslash r_{Q}\left(I_{i+1}\right)$ for all $i$, which contradicts the hypothesis that $Q$ is right Artinian.

PROPOSITION 4.5. Let $R$ be a ring and let $G$ be a group. If $R G$ has a right Artinian right quotient ring, then so does $R$.

PrOOF. Using Lemmas 3.2 and 4.4, we see that $\Delta^{+}(G)$ is finite so in view of Lemma 4.3, we may assume that $\Delta^{+}(G)=1$. Let $N$ be the nilradical of $R$ and let $Q$ be the right Artinian right quotient ring of $R G$. Since $R G$ is right Goldie (see p. 8 of [1]), so is $R$ and hence $N$ is nilpotent by Theorem 1.35 of [1]. Therefore if $J=N Q$, then $J$ is a nilpotent ideal of $Q$ (use Theorem 1.31 of [1]) and hence $J \cap R G$ is a nilpotent ideal of $R G$. Since $(R / N) G \cong R G / N R G$, it follows from Theorem 2 on p. 37 of [11] that $J \cap R G=N R G$ and we deduce that $Q / J$ is a right Artinian right quotient ring for $(R / N) G$. This shows that $R / N$ is right Goldie. If $\rho_{R}(R)=\infty$ then $\rho_{R}\left(N^{i-1} / N^{i}\right)=\infty$ for some $i \in \mathbf{P}$ by Lemma 2.4(a), and it follows easily that $\rho_{R G}\left(N^{i-1} R G / N^{i} R G\right)=\infty$. Therefore $\rho_{R G}(R G)=\infty$ by Lemma 2.4(a). On the other hand $\rho_{R G}\left(J^{l-1} \cap R G / J^{l} \cap R G\right)<\infty$ for all $l \in \mathbf{P}$, hence $\rho_{R G}(R G)<\infty$ by Lemma 2.4(a) and we have a contradiction. Thus $\rho_{R}(R)<\infty$, so by Lemma 2.5 it will be sufficient to prove that if $\alpha \in R$ is such that its image $\bar{\alpha}$ in $R / N$ is a nonzero divisor, then $\alpha$ is a nonzero divisor. But

$\bar{\alpha}$ is a nonzero divisor in $R / N$

$\Rightarrow \bar{\alpha}$ is a nonzero divisor in $(R / N) G$

$\Rightarrow \bar{\alpha}$ is invertible in $Q / J$

$\Rightarrow \alpha$ is invertible in $Q$ because $J$ is nilpotent

$\Rightarrow \alpha$ is a nonzero divisor in $R$

as required.

LEMMA 4.6. Let $R$ be a simple Artinian ring and let $G \in \mathscr{C}$ with $\Delta^{+}(G)=1$. If $R G$ has a right Artinian right quotient ring, then the finite subgroups of $G$ have bounded order.

Proof. Since $\Delta^{+}(G)=1$, we see that $R G$ is prime by Theorem 2 on p. 37 of [11], so if $Q$ is the right Artinian right quotient ring of $R G$, then $Q=M_{n}(D)$ for some $n \in \mathbf{P}$ and division ring $D$. 
The result will be proved by transfinite induction, so by Lemma 3.1 choose the least ordinal $\alpha$ such that $G \in \mathscr{X}_{\alpha}$, and assume that the result is true whenever $G \in \mathscr{X}_{\beta}$ and $\beta<\alpha$. Let $F \in \mathscr{F}(G)$ and write $e=\sum_{f \in F} f$.

Now $\alpha$ cannot be a limit ordinal, and the result is clearly true if $\alpha=0$. Therefore we may assume that $\alpha=\gamma+1$ for some ordinal $\gamma$. First suppose $G \in L \mathscr{Z}_{\gamma}$. Since $R G$ is a subring of the right Artinian ring $Q$, we see from Lemma 4.4 that $G$ has no infinite locally finite subgroup and so we can use Lemma 3.3. Thus there exists $P \leq G$ such that $P \supseteq F, P \in \mathscr{X}_{\gamma}$ and $\Delta^{+}(P)=1$. We now apply Lemma 4.1 to deduce that $R P$ has a simple Artinian right quotient ring $W$, and that $(e W)^{|F|} \cong W$. Therefore $(e Q)^{|F|} \cong Q$ and hence $|F| \leq n$ as required.

Now suppose there exists $H \triangleleft G$ such that $H \in L \mathscr{X}_{\gamma}$ ands $G / H \in \mathscr{B}$. By the previous paragraph, the finite subgroups of $H$ have bounded order and it follows that the finite subgroups of $G$ have bounded order.

PROPOSITION 4.7. Let $R$ be a ring and let $G \in \mathscr{C}$. If $R G$ has a right Artinian right quotient ring, then the finite subgroups of $G$ have bounded order.

Proof. Since $\Delta^{+}(G)$ is finite by Lemmas 4.4 and 3.2, application of Lemma 4.3 shows that we may assume that $\Delta^{+}(G)=1$. In view of Proposition 4.5, we may also assume that $R$ is right Artinian. Let $Q$ be a right Artinian right quotient ring for $R G$, let $J$ be the radical of $Q$, and let $N$ be the radical of $R$. Since $R G / N R G \cong(R / N) G$, it follows from Theorem 2 on p. 37 of [11] that $N R G$ is the nilradical of $R G$. Using Theorem 1.31 of [1], we see that $N Q$ is a nilpotent ideal of $Q$, hence $N Q \subseteq J$ and we deduce that $J \cap R G=N R G$. Therefore $Q / J$ is an Artinian right quotient ring for $(R / N) G$. Furthermore, $R / N$ is a direct sum of simple Artinian rings, thus we may assume that $R$ is a simple Artinian ring and now the result follows from Lemma 4.6.

Theorem 1.2 now follows from Propositions 4.2, 4.5, and 4.7, and Theorem 1.3 from Lemma 4.1(iv).

5. Semiprime crossed products. Suppose $G \in \mathscr{C}, R$ is a semisimple Artinian ring, $R * G$ is a crossed product, $\Delta^{+}(G)=1$ and the finite subgroups of $G$ have bounded order. Then $R=R_{1} \oplus \cdots \oplus R_{m}$ where the $R_{i}$ are simple Artinian rings, and $G$ permutes the $R_{i}$ by conjugation. By renumbering if necessary, we may assume that $\left\{R_{1}, \ldots, R_{t}\right\}$ is a set of orbit representatives for this action. Let $G_{i}$ be the stabilizer of $R_{i}$, and write $n_{i}=G: G_{i}$. By Clifford's theorem,

$$
R * G=\bigoplus_{i=1}^{t} M_{n_{i}}\left(R_{i} * G_{i}\right) .
$$

Since $\Delta^{+}\left(G_{i}\right)=1$ and $R_{i}$ is simple Artinian, it follows from Lemma 4.1 that $R_{i} * G_{i}$ has a right quotient ring $M_{l_{i}}\left(D_{i}\right)$ where $D_{i}$ is a division ring and

$$
\begin{array}{r}
l_{i}=1 . c . m .\left\{|F| / \rho_{R_{i}}[M] \mid F \in \mathscr{F}\left(G_{i}\right) \text { and } M \neq 0\right. \text { is a finitely generated } \\
\left.R_{i} * F \text {-module }\right\} .
\end{array}
$$

Therefore $R * G$ has a quotient ring isomorphic to $\bigoplus_{i=1}^{t} M_{n_{i} l_{i}}\left(D_{i}\right)$.

Suppose $H \in \mathscr{C}$, the finite subgroups of $H$ have bounded order, and $k$ is a division ring of characteristic zero. Then we can apply the above discussion to $k * H$ because if $N=\Delta^{+}(H)$, then $k * N$ is semisimple Artinian (Theorem 5 on p. 
31 of [11] and Lemma 3.2), $\Delta^{+}(H / N)=1$ and $k * H \cong(k * N) * H / N$ (pp. 29-30 of $[11])$.

NOTE ADDED IN PROOF. An explicit reference for Lemma 2.5 is Theorem (4) of J. A. Beachy, Rings with finite reduced rank, Comm. Algebra 10 (1982), 1517-1536.

\section{REFERENCES}

1. A. W. Chatters and C. R. Hajarnavis, Rings with chain conditions, Research Notes in Math., no. 44, Pitman, Boston, Mass., 1980.

2. K. A. Brown, On zero divisors in group rings, Bull. London Math. Soc. 8 (1976), 251-256.

3. __ Artinian quotient rings of group rings, J. Algebra 49 (1977), 63-80.

4. C. Chou, Elementary amenable groups, Illinois J. Math. 24 (1980), 396-407.

5. E. C. Dade, Group-graded rings and modules, Math. Z. 174 (1980), 241-262.

6. D. R. Farkas and R. L. Snider, $K_{0}$ and Noetherian group rings, J. Algebra 42 (1976), 192-198.

7. J. A. Moody, Ph.D. Thesis, Columbia University, 1986.

8. __ Induction theorems for infinite groups, Bull. Amer. Math. Soc. 17 (1987), 113-116.

9.

10. D. S. Passman, Infinite group rings, Dekker, New York, 1971.

11. __ Group rings, crossed products and Galois theory, CBMS Regional Conf. Ser. in Math., no. 64, Amer. Math. Soc., Providence, R. I., 1986.

12. S. Rosset, The Goldie rank of virtually polycyclic groups, The Brauer Group, Lecture Notes in Math., vol. 844, Springer-Verlag, Berlin, 1981, pp. 35-45.

Department of Mathematics, St. John's College, Cambridge CB2 ITP, EngLAND

Department of Mathematics, Virginia Polytechnic Institute and State UNIVERSity, BlaCKSBURG, Virginia 24061-0123 (Current address of P. A. Linnell) 78712

Current address: (P. H. Kropholler and J. A. Moody): Department of Mathematics, Queen Mary College, Mile End Road, London E1 4N5, England 